\title{
Cardiac innervation imaging as a risk stratification tool for potential device therapy candidates
}

\author{
Rudolf A. Werner, MD \\ a Division of Nuclear Medicine and Molecular Imaging, The Russell H. Morgan Department of \\ Radiology and Radiological Science, Johns Hopkins University School of Medicine, Baltimore, \\ MD \\ b Department of Nuclear Medicine/Comprehensive Heart Failure Center, University Hospital \\ Würzburg, Würzburg, Germany
}

Received Sep 19, 2018; accepted Sep 20, 2018

doi: $10.1007 / \mathrm{s} 12350-018-01475-0$

As a scintigraphic approach evaluating cardiac nerve integrity, ${ }^{123}$ I-metaiodobenzylguanidine ( ${ }^{123}$ I-mIBG) has been recently Food and Drug Administration approved. A great deal of progress has been made by the prospective ADMIRE-HF trial, which primarily demonstrated the association of denervated myocardium assessed by ${ }^{123}$ I-mIBG and cardiac events. However, apart from risk stratification, myocardial nerve function evaluated by molecular imaging should also be expanded to other clinical contexts, in particular to guide the referring cardiologist in selecting appropriate candidates for specific therapeutic interventions. In the present issue of the Journal of Nuclear Cardiology ${ }^{\circledR}$, the use of ${ }^{123}$ I-mIBG for identifying cardiomyopathy patients, which would most likely not benefit from ICD due to low risk of arrhythmias, is described. If we aim to deliver on the promise of cardiac innervation imaging as a powerful tool for risk stratification in a manner similar to nuclear oncology, studies such as the one reviewed here may imply an important step to lay the proper groundwork for a more widespread adoption in clinical practice.

Key Words: Heart failure • cardiomyopathy • SPECT $・$ cardiac innervation • molecular imaging • ICD

See related article, pp. 1787-1797

Alterations of the myocardial sympathetic nervous system (SNS) in Heart Failure (HF) patients are primarily driven by cardiac norepinephrine (NE) spillover, which in turn provokes severe damage of

Reprint requests: Rudolf A. Werner, MD, Division of Nuclear Medicine and Molecular Imaging, The Russell H. Morgan Department of Radiology and Radiological Science, Johns Hopkins University School of Medicine, 601 N. Caroline St. JHOC 3230, Baltimore, MD 21287; werner_r1@ukw.de; rwerner3@jhmi.edu J Nucl Cardiol 2020;27:1798-801.

1071-3581/\$34.00

Copyright (C) 2018 American Society of Nuclear Cardiology. cardiomyocytes, in particular by AMP-mediated calcium overload. ${ }^{1,2}$ Notably, such a continuous NE oversupply also triggers remodeling of the left ventricle or the development of hypertensive left ventricular hypertrophy. ${ }^{3}$ On a subcellular level, cardiac alterations of the SNS are reflected by impaired function of the NE transporter (uptake-1 mechanism) and by reduced plasma clearance of NE in the synaptic cleft. ${ }^{1,4}$ As a physiological rationale, NE is stored inside presynaptic vesicles and if a firing impulse has arrived at the nerve terminal, NE is released into the synaptic gap and provokes further downstream cascades at postsynaptic Badrenoreceptors to achieve neurotransmission. After completing its primary task at the post-synapse, NE has to undergo a recycling mechanism via the uptake- 1 and is stored in storage vesicles for potential re-use. Notably, as a non-invasive mean, cardiac SNS radiotracers using 


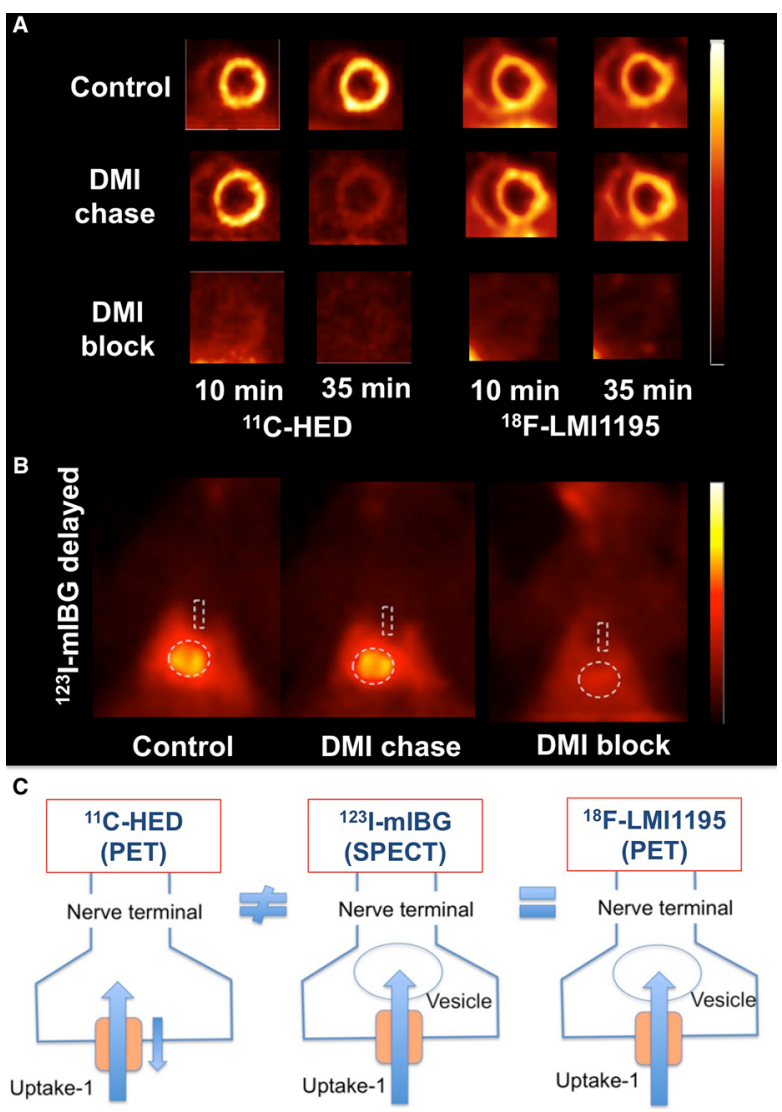

Figure 1. Cardiac nerve radiotracer handling at the sympathetic nerve terminal. (A) Representative short-axis images of in-vivo rabbit cardiac PET imaging. Desipramine (DMI) chase (i.e., DMI administered after tracer delivery) enhanced ${ }^{11} \mathrm{C}$ Hydroxyephedrine $\left({ }^{11} \mathrm{C}\right.$-HED) tracer washout, while ${ }^{18} \mathrm{~F}$ LMI1195 washout was not impacted. (B) Results of in-vivo rabbit ${ }^{123}$ I-metaiodobenzylguanidine $\quad\left({ }^{123} \mathrm{I}-\mathrm{mIBG}\right)$ planar scintigraphy of the chest. A high affinity of ${ }^{123}$ I-mIBG to neuronal uptake-1 was confirmed by the DMI blocking protocol (DMI injection prior to radiotracer administration). Similar to ${ }^{18} \mathrm{~F}$-LMI1195, DMI chase did not influence cardiac distribution of ${ }^{123} \mathrm{I}-\mathrm{mIBG}$. Of note, both radiotracers share the similar underlying benzylguanidine structure. Dotted lines indicate regions of interest in both heart and mediastinum. $(\mathbf{C})$ Schematic figure of radiotracer handling at the sympathetic nerve terminal. In light of those findings, distinct radiotracer characteristics has been proven: After being taken up via uptake-1, ${ }^{123}$ I-mIBG and ${ }^{18}$ F-LMI1195 are stably stored at nerve terminals and thus, closely mimic their physiological counterpart norepinephrine. This is in contradistinction to ${ }^{11} \mathrm{C}$ HED, which primarily undergoes a continuous cycle of uptake and release at the nerve terminal (i.e., continuous re-uptake via uptake-1). Altogether, such preclinical experiments using dedicated animal platforms may lay the proper groundwork for risk stratification studies in a clinical environment, such as the one reviewed in the present issue of $J N C$. Modified from Werner et al $^{10}$ (C) by the Society of Nuclear Medicine and Molecular Imaging, Inc. either single photon emission computed tomography (SPECT) or positron emission tomography (PET) technology, have been advocated to share similar pathways like physiological NE. Thus, impaired cardiac nerve integrity is either reflected by increased radiotracer washout or a significant decrease in radiotracer uptake. Taken together, those cardiac nerve imaging agents may allow for a precise assessment of sympathetic neurotransmission in the failing heart. ${ }^{5,6}$

As a a scintigraphic approach, ${ }^{123}$ I-metaiodobenzylguanidine $\left({ }^{123} \mathrm{I}-\mathrm{mIBG}\right)$ has been recently Food and Drug Administration approved and is extensively used in clinical practice. ${ }^{7-9}$ However, in recent years, the field is expanding, in particular as cardiac neuronal PET imaging agents may offer an even more thorough evaluation of cardiac nerve integrity, mainly due an increased spatio-temporal resolution and the capability of quantification. Thus, PET SNS radiotracer may reveal further underlying characteristics of myocardial nerve function, such as regional analysis of different "myocardial areas of interest" (e.g., infarct area, border zone, or remote myocardium). Among those PET imaging agents, ${ }^{11} \mathrm{C}$-Hydroxyephedrine $\left({ }^{11} \mathrm{C}\right.$-HED $)$ is currently at the forefront for assessing myocardial innervation non-invasively, but expenses for purchase, its short half-life of 20 minutes, and the maintenance of costly on-site cyclotrons, are considerations for practitioners as to what extent such a radiopharmaceuticals can be employed in the clinic. Notably, introduction of novel ${ }^{18} \mathrm{~F}$ SNS imaging agents, such as ${ }^{18} \mathrm{~F}$-LMI1195, may overcome these limitations: the long physical halflife of $110 \mathrm{~min}$ allows for the use of delivery systems from central cyclotron facilities or even distribution by commercial vendors, which has proven to be cost-effective for the most commonly used imaging agent in nuclear oncology, 2-deoxy-2 ${ }^{18}$ F-fluoro-D-glucose. ${ }^{5}$ However, if such cardiac nerve SPECT/PET probes should be one day become routinely available in the clinic to identify patients at highest risk for cardiac events, a precise understanding of the exact catecholamine radiotracer handling of such "false neurotransmitters" at the nerve terminal is indispensable. Thus, efforts in recent years aimed to provide deeper insights of cardiac retention kinetics on a subcellular level, which may be fundamental for a proper interpretation of imaging results. An extensive body of evidence has confirmed the high affinity of ${ }^{123} \mathrm{I}-\mathrm{mIBG}$ to the neuronal uptake-1, which forms the backbone of SNS imaging of the heart: a significant decrease of the heart-to-mediastinum ratio (HMR) after pre-treatment with the potent uptake-1 blocker desipramine (DMI) in rabbit myocardium was proven. ${ }^{7}$ In an in-vivo tripletracer comparison in healthy rabbits using ${ }^{123} \mathrm{I}-\mathrm{mIBG}$, ${ }^{11} \mathrm{C}$-HED and ${ }^{18} \mathrm{~F}$-LMI1195, a selective uptake- 1 
blockage using DMI was reported for all three investigated imaging agents. Notably, distinct radiotracer characteristics could be proven by using a DMI chase protocol, i.e., DMI injected after tracer delivery. Sharing the same benzylguanidine structure, retention kinetics of ${ }^{123} \mathrm{I}$-mIBG and ${ }^{18} \mathrm{~F}$-LMI1195 remained stable, while ${ }^{11} \mathrm{C}$-HED demonstrated increased radiotracer washout (Figure 1A, B). Thus, after being taken up via uptake-1, ${ }^{123}$ I-mIBG and ${ }^{18} \mathrm{~F}$-LMI1195 are stably stored inside presynaptic vesicles and thus, closely mimick their physiological counterpart NE. This is in contradistinction to ${ }^{11} \mathrm{C}-\mathrm{HED}$, which primarily undergoes a continuous cycle of uptake and release at the nerve terminal (Figure 1C). ${ }^{10}$ Those distinct radiotracer characteristics were further corroborated in NE-expressing, vesicle-rich PC12 and vesicle-poor SK-N-SH cell lines: After using stimulants for storage vesicle turnover (reserpine, high potassium chloride), both ${ }^{123} \mathrm{I}-\mathrm{mIBG}$ and ${ }^{18}$ F-LMI1195 demonstrated enhanced washout from PC12 cells, while retention for those cardiac SNS imaging agents remained stable in SK-N-SH cells. ${ }^{11}$

After laying the proper groundwork of an exact understanding of radiotracer handling at the sympathetic nerve terminal, an imaging interpretor can have confidence that those imaging probes precisely reflect cardiac sympathetic neurotransmission and thus, can be truly described as "false neurotransmitters". In this regard, the next step are clinical applications of such imaging agents on a broader perspective, in particular for risk stratification of patients which are at highest risk to suffer from lethal cardiac events. A great deal of progress has been made by the prospective "AdreView Myocardial Imaging for Risk Evaluation in Heart Failure (ADMIRE-HF)" trial, which primarily demonstrated the association of denervated myocardium evaluated by ${ }^{123}$ I-mIBG HMR and HF-related events, independent of other common clinical parameters, such as left ventricular ejection fraction (LVEF). ${ }^{12}$ Notably, in a sophisticated approach, Nakajima et al created mortality risk charts using ${ }^{123}$ I-mIBG derived HMR and clinical parameters (LVEF, New York Heart Association Class) and suggested 2- and 5-year mortality risk estimations. ${ }^{13}$ However, if ${ }^{123} \mathrm{I}$-mIBG SPECT or other cardiac neuronal PET radiotracers become one day more routinely available in the clinic, those cardiac nerve radiotracers must be applicable to a wide array of particular clinical scenarios. Thus, apart from risk stratification for HF-related events, cardiac nerve integrity assessed by ${ }^{123} \mathrm{I}$-mIBG should also be expanded to other clinical contexts, in particular to guide the referring cardiologist in selecting the appropriate candidate for specific therapeutic interventions. In the present issue of the $J N C$, the use of ${ }^{123} \mathrm{I}$-mIBG for identifying cardiomyopathy patients, which would most likely not benefit from ICD due to low risk of arrhythmias, is described. ICD shocks for ventricular tachyarrhythmias play a crucial role for secondary prevention of sudden cardiac death (relative risk reduction, $20 \%$ ). Albeit the documented shock frequency is described inconsistently among different studies, a considerable high number of subjects still receive an inappropriate shock $(11.5 \%)$, which in turn results in deterioration of health-related quality of life or even fatal ventricular arrhythmia. ${ }^{14,15}$ Such a malfunction is in contradistinction to $65 \%$ of the patients, which will never have an appropriate ICD discharge. ${ }^{5,16,17}$ Spearheaded by Scrima et al in a multicentric setting, 81 patients suffering from either ischemic or non-ischemic cardiomyopathy and a LVEF $\leq 35 \%$ were enrolled. None of the included patients had any previous history of malignant ventricular arrhythmias. Using the wellestablished HMR cutoff of 1.6 on delayed ${ }^{123} \mathrm{I}-\mathrm{mIBG}$ derived planar images, 54 subjects were assigned to the HMR group beyond and 27 patients to the group above the cutoff. After a mean follow-up of 13.3 $( \pm 9.7)$ months, the primary end-point (malignant arrhythmias) occured in 13/81 (16\%), and all of those patients had been allocated to the high-risk group according to planar scintigraphic assessment (late HMR $<1.6$ ), while no such events occured in the low-risk group (HMR > 1.6). In addition, $7 / 81(8.6 \%)$ patients died of cardiac events, with the majority $(6 / 7,85.7 \%)$ having previously assigned to the high-risk by cardiac nerve SPECT. Notably, an HMR $\geq 1.6$ had a considerable high negative predictive value for cardiac death and worsening HF (up to $96 \%$ ). The findings of Scrima et al are in line with a previous performed post-hoc analysis which demonstrated that the combination of HMR and LVEF are associated with freedom of appropriate ICD therapy. ${ }^{17}$ Taken together, ${ }^{123}$ I-mIBG might be helpful in excluding those patients who might not benefit from ICD and thus, may significantly contribute to an improved guidance of such highly specific and costly therapeutic interventions. Thus, one might hypothesize that measuring cardiac nerve integrity with such non-invasive metrics may outperform current established criteria for patient selection, e.g., LVEF or QRS duration. ${ }^{18}$ However, larger clinical trials, preferably in a prospective setting, are warranted to corroborate those preliminary findings and to further establish precision cardiology through molecular imaging, e.g., by the use of cardiac neuronal PET radiotracers.

Compared to nuclear cardiology, molecular imaging has been extensively used for risk stratification in a large variety of oncological applications. Aiming at tailored medical treatment for different tumor entities, efforts in nuclear oncology aimed to provide decision-support in 
disease management, e.g., by identifying the prognostic capability of baseline somatostatin-receptor PET in neuroendocrine tumor patients scheduled for peptide receptor radionuclide therapy or the prognostic performance of prostate-specific membrane antigen PET in prostate cancer patients. ${ }^{19,20}$ Thus, if we aim to deliver on the promise of cardiac innervation imaging as a powerful tool for risk stratification among HF patients in a manner similar to nuclear oncology, studies such as the one reviewed here may imply an important step to lay the proper groundwork for a more widespread adoption in clinical practice.

\section{Disclosures}

$R A W$ has received funding from the European Union's Horizon 2020 research and innovation program under the Marie Sklodowska-Curie Grant Agreement No. 701983.

\section{References}

1. Liang CS. Cardiac sympathetic nerve terminal function in congestive heart failure. Acta Pharmacol Sin 2007;28:921-7.

2. Mann DL, Kent RL, Parsons B, Cooper GT. Adrenergic effects on the biology of the adult mammalian cardiocyte. Circulation 1992;85:790-804.

3. Schlaich M, Kaye DM, Lambert E, Sommerville M, Socratous F, Esler MD. Relation between cardiac sympathetic activity and hypertensive left ventricular hypertrophy. Circulation 2003; 108:560-5.

4. Parati G, Esler M. The human sympathetic nervous system: Its relevance in hypertension and heart failure. Eur Heart $\mathrm{J}$ 2012;33:1058-66.

5. Werner RA, Chen X, Hirano M, Rowe SP, Lapa C, Javadi MS, et al. SPECT vs. PET in cardiac innervation imaging: Clash of the titans. Clin Transl Imaging 2018;6:293-303.

6. Thackeray JT, Bengel FM. PET imaging of the autonomic nervous system. Q J Nucl Med Mol Imaging 2016;60:362-82.

7. Werner RA, Kobayashi R, Javadi MS, Kock Z, Wakabayashi H, Unterecker $S$, et al. Impact of novel antidepressants on cardiac (123)I-metaiodobenzylguanidine uptake: Experimental studies on SK-N-SH cells and healthy rabbits. J Nucl Med 2018;59:1099103.

8. Nakajima K, Nakata T. Cardiac 123I-MIBG imaging for clinical decision making: 22-year experience in Japan. J Nucl Med 2015;56:11S-9S
9. Henzlova MJ, Duvall WL, Einstein AJ, Travin MI, Verberne HJ. ASNC imaging guidelines for SPECT nuclear cardiology procedures: Stress, protocols, and tracers. J Nucl Cardiol 2016;23:60639.

10. Werner RA, Rischpler C, Onthank D, Lapa C, Robinson S, Samnick S, et al. Retention kinetics of the 18F-labeled sympathetic nerve PET tracer LMI1195: Comparison with 11Chydroxyephedrine and 123I-MIBG. J Nucl Med 2015;56:1429-33.

11. Chen X, Werner RA, Lapa C, Nose N, Hirano M, Javadi MS, et al. Subcellular storage and release mode of the novel (18)F-labeled sympathetic nerve PET tracer LMI1195. EJNMMI Res 2018;8:12.

12. Jacobson AF, Senior R, Cerqueira MD, Wong ND, Thomas GS, Lopez VA, et al. Myocardial iodine-123 meta-iodobenzylguanidine imaging and cardiac events in heart failure. Results of the prospective ADMIRE-HF (AdreView Myocardial Imaging for Risk Evaluation in Heart Failure) study. J Am Coll Cardiol 2010;55:2212-21.

13. Nakajima K, Nakata T, Matsuo S, Jacobson AF. Creation of mortality risk charts using $123 \mathrm{I}$ meta-iodobenzylguanidine heartto-mediastinum ratio in patients with heart failure: 2- and 5-year risk models. Eur Heart J Cardiovasc Imaging 2016;17:1138-45.

14. Daubert JP, Zareba W, Cannom DS, McNitt S, Rosero SZ, Wang $\mathrm{P}$, et al. Inappropriate implantable cardioverter-defibrillator shocks in MADIT II: Frequency, mechanisms, predictors, and survival impact. J Am Coll Cardiol 2008;51:1357-65.

15. Vollmann D, Luthje L, Vonhof S, Unterberg C. Inappropriate therapy and fatal proarrhythmia by an implantable cardioverterdefibrillator. Heart Rhythm 2005;2:307-9.

16. Moss AJ, Greenberg H, Case RB, Zareba W, Hall WJ, Brown MW, et al. Long-term clinical course of patients after termination of ventricular tachyarrhythmia by an implanted defibrillator. Circulation 2004;110:3760-5.

17. Verschure DO, de Groot JR, Mirzaei S, Gheysens O, Nakajima K, van Eck-Smit BLF, et al. Cardiac (123)I-mIBG scintigraphy is associated with freedom of appropriate ICD therapy in stable chronic heart failure patients. Int J Cardiol 2017;248:403-8.

18. Brignole M, Auricchio A, Baron-Esquivias G, Bordachar P, Boriani G, Breithardt OA, et al. 2013 ESC Guidelines on cardiac pacing and cardiac resynchronization therapy: the Task Force on cardiac pacing and resynchronization therapy of the European Society of Cardiology (ESC). Developed in collaboration with the European Heart Rhythm Association (EHRA). Eur Heart J 2013;34:2281-329.

19. Werner RA, Ilhan H, Lehner S, Papp L, Zsoter N, Schatka I, et al. Pre-therapy somatostatin receptor-based heterogeneity predicts overall survival in pancreatic neuroendocrine tumor patients undergoing peptide receptor radionuclide therapy. Mol Imaging Biol 2018. https://doi.org/10.1007/s11307-018-1261-4.

20. Kletting P, Thieme A, Eberhardt N, Rinscheid A, D'Alessandria C, Allmann J, et al. Modeling and predicting tumor response in radioligand therapy. J Nucl Med 2018. https://doi.org/10.2967/jn umed.118.210377. 